\title{
BEHNKE-STEIN THEOREM ON COMPLEX SPACES WITH SINGULARITIES
}

\author{
M. COLTOIU AND A. SILVA
}

\section{§0. Introduction}

Let $X$ be a non-compact Riemann surface and $D \subset X$ an open subset. By a classical result due to Behnke and Stein [2] $D$ is Runge in $X$ (i.e. the restriction map $\mathscr{O}(X) \rightarrow \mathscr{O}(D)$ has dense image) iff $X \backslash D$ has no compact connected components. In other words the obstruction to holomorphic approximation is purely topological. This result has been generalized to 1-dimensional Stein spaces by Mihalache in [11].

Let now $X$ be a connected non-compact complex manifold of dimension $n$ and $D \subset X$ an open subset. The open subset $D$ is said to be $n$-Runge in $X$ if for every compact set $K \subset D$ there exists an $n$-convex exhaustion function $\varphi: X \rightarrow \mathbf{R}$ such that $K \subset\{\varphi<o)$ and $\{\varphi \leq o\} \subset D$. Clearly this notion extends the classical one in dimension 1 and, by the results of Andreotti and Grauert [1], if $D$ is $n$-Runge in $X$ then the natural restriction map $H^{n-1}(X, \mathscr{F}) \rightarrow H^{n-1}(D, \mathscr{F})$ has dense image for every $\mathscr{F} \in \operatorname{Coh}(X)$. Also from the results of Greene and $\mathrm{Wu}$ [7] it follows that $D$ is $n$-Runge in $X$ iff $X \backslash D$ has no compact connected components (see also [10]).

The main purpose of this paper is to generalize the above results to complex spaces with singularities. More precisely we prove:

THEOREM. Let $X$ be a complex space of pure dimension $n$ with no compact irreducible components and $D \subset X$ an open subset. Then the following conditions are equivalent:

1) $D$ is $n$-Runge in $X$

2) For every $\mathscr{F} \in \operatorname{Coh}(X)$ the restriction map $H^{n-1}(X, \mathscr{F}) \rightarrow H^{n-1}(D, \mathscr{F})$ has dense image

3) If $\Omega_{X}^{n}$ is the canonical sheaf of $X$ then the restriction map $H^{n-1}\left(X, \Omega_{X}^{n}\right) \rightarrow$ $H^{n-1}\left(D, \Omega_{X}^{n}\right)$ has dense image.

Received March 28, 1994. 
4) The natural map $H_{2 n-1}(D, \mathbf{C}) \rightarrow H_{2 n-1}(X, \mathbf{C})$ is injective.

5) $X \backslash D$ has no compact irreducible components.

Let us remark that for $D=\phi$ (empty set) this Theorem implies that $X$ is $n$-complete, a well-known result due to Ohsawa [13].

Our proof of the above theorem uses resolution of singularities [8], a theorem of Diederich and Fornaess [5] on the approximation of $q$-convex functions with corners, and a result due to Takegoshi [19] (see also Ohsawa [14]) on the vanishing of $R^{2} \pi_{*} \Omega_{\tilde{X}}^{n}$ where $\pi: \tilde{X} \rightarrow X$ is a resolution of singularities for the $n$-dimensional complex space $X$ and $\Omega_{\tilde{X}}^{n}$ is the sheaf of germs of holomorphic differential $n$-forms on $\tilde{X}$.

\section{§1. Preliminaries and definitions}

All complex spaces considered in this paper are assumed to be reduced and with countable topology.

It is known [8] that any complex space $X$ admits a resolution of singularities $\pi: \tilde{X} \rightarrow X$, i.e. $\tilde{X}$ is a complex manifold and $\pi$ is a proper modification such that the induced map $\pi^{-1}(\operatorname{Reg}(X)) \rightarrow \operatorname{Reg}(X)$ is an isomorphism. Let $n=\operatorname{dim}(X)$ and let $\pi: \tilde{X} \rightarrow X$ be a resolution of singularities. If $\Omega_{\tilde{X}}^{n}$ denotes the sheaf of germs of holomorphic differential $n$-forms on $\tilde{X}$ then we define the canonical sheaf $\Omega_{X}^{n}$ of $X$ to be the 0 -direct image $\pi_{*} \Omega_{\tilde{X}}^{n}$. By Grauert's coherence theorem $\Omega_{X}^{n}$ is a coherent sheaf on $X$. Moreover, this definition does not depend on the chosen resolution of singularities [6]. When $X$ is normal, an equivalent definition for $\Omega_{X}^{n}$ is the following: Let $S \subset X$ be the singular locus of $X$ and for any open set $U \subset X$ define $\Gamma(U)=\left\{\right.$ the holomorphic $n$-fomrs $\varphi$ on $U \backslash S$ such that $\left.\int_{U \backslash S} \varphi \wedge \bar{\varphi}<\infty\right\}$. Then the sheaf associated to the presheaf $\{\Gamma(U)\}$ is exactly $\Omega_{X}^{n}[6]$.

One has the following result due to Takegoshi [19] (see also Ohsawa [14]):

Theorem 1. If $X$ is a comlex space of pure dimension $n$ and $\pi: \tilde{X} \rightarrow X$ is a resolution of singularities then $R^{i} \pi_{*} \Omega_{\tilde{X}}^{n}=0$ for $i \geq 1$.

Let now $X$ be a complex space and $D \subset X$ an open subset. Let $\nu: \hat{X} \rightarrow X$ be the normalization of $X$ and $\hat{D}=\nu^{-1}(D)$. We say that $X \backslash D$ has nó compact irreducible components iff $\hat{X} \backslash \hat{D}$ has no compact connected components.

If $U$ is an open subset in $\mathbf{C}^{n}$, a function $\varphi \in C^{\infty}(U, \mathbf{R})$ is called $q$-convex iff the Levi form $L(\varphi)$ has at least $(n-q+1)$ positive $(>0)$ eigenvalues at any 
point of $U$. Using local embeddings this notion can be easily extended to complex spaces [1]. A complex space $X$ is called $q$-complete iff there exists a $q$-convex function $\varphi: X \rightarrow \mathbf{R}$ which is an exhaustion function on $X$, i.e. $X_{c}=\{\varphi<c\} \subset \subset$ $X$ for any $c \in \mathbf{R}$.

If $X$ is a complex space and $D \subset X$ is an open subset we say that $D$ is $q$-Runge in $X$ iff the following holds: for every compact set $K \subset D$ there exists a $q$-convex function $\varphi$ on $X$ which is an exhaustion function on $X$ and such that $K \subset\{\varphi<0\}$ and $\{\varphi \leqslant 0\} \subset D$. It follows from this definition that $X$ is assumed to be $q$-complete.

One has the following topological property for $q$-Runge domains [20]: If $X$ is an $n$-dimensional complex space and $D \subset X$ is $q$-Runge then the relative homology groups $H_{\imath}(X, D ; \mathbf{C})$ vanish for every $i \geqslant n+q$.

In particular it follows:

Corollary 1. Let $X$ be an $n$-dimensional complex space and $D \subset X$ an $n$-Runge open set. Then the natural map $H_{2 n-1}(D, \mathbf{C}) \rightarrow H_{2 n-1}(X, \mathbf{C})$ is injective.

Let us now recall some basic facts about $q$-convex functions with corners [5]. If $X$ is a complex space we denote by $F_{q}(X)$ the $q$-convex functions with corners on $X$ i.e. those continuous functions $f$ on $X$ such that for any point $x \in X$ there is an open neighbourhood $U=U(x)$ of $x$ and finitely many $q$-convex functions $f_{1}, \ldots, f_{s}$ on $U$ such that $\left.f\right|_{U}=\max \left(f_{1}, \ldots, f_{s}\right)$.

In [5] the following result is proved:

THEOREM 2. Let $X$ be a complex space of dimension $n, f \in F_{q}(X), 1 \leq q \leq n$, and $\eta>0$ a continuous function on $X$. Then there exists an $\tilde{q}$-convex function $\tilde{f}$ on $X$, where $\tilde{q}=n-[n / q]+1$, such that $|f-\tilde{f}|<\eta$ on $X$.

In particular, when $q=n$ we get from the above theorem the following:

Corollary 2. Let $X$ be a complex space of dimension $n, f \in F_{n}(X)$, and $\eta>0$ a continuous function on $X$. Then there exists an $n$-convex function $\tilde{f}$ on $X$, such that $|f-\tilde{f}|<\eta$ on $X$.

We shall need also the following result from [3]:

Lemma 1. Let $X$ be a complex space, $A \subset X$ a closed analytic subset, $f \in$ $F_{q}(A)$ and $\eta>0$ a continuous function on $A$. Then there exists an open neighbourhood $V$ of $A$ and $f \in F_{q}(V)$ such that $|f-\tilde{f}|<\eta$ on $A$. 


\section{§2. Proof of the Theorem}

Before going into the proof of the Theorem we shall establish some lemmas.

Lemma 2. Let $X$ be a complex manifold of pure dimension $n$ with no compact connected components and $K \subset X$ a compact subset such that $X \backslash K$ has no relatively compact connected components. Then for every open neighbourhood $U$ of $K$ there exists an $n$-convex exhaustion function $\varphi: X \rightarrow \mathbf{R}$ with $K \subset\{\varphi<0\}$ and $\{\varphi \leqslant 0\} \subset U$.

Proof. Clearly we may assume $X$ connected. If $h$ is any hermitian metric on $X$ then it induces a riemannian metric on $X_{\text {real }}$ and by the results in [7] there is a strongly sub-harmonic function $\varphi$ on $X_{\text {real }}$, with respect to the riemannian induced metric, such that $\varphi$ is an exhaustion function on $X, K \subset\{\varphi<0\}$ and $\{\varphi \leqslant 0\} \subset$ $U$. Clearly $\varphi$ is $n$-convex with respect to the complex structure of $X$.

Lemma 3. Let $X$ be a complex manifold of pure dimension $n$ with no compact connected components, $\Omega^{n}$ the sheaf of germs of holomorphic differential $n$-forms on $X$ and $D \subset X$ an open subset. If the restriction map $H^{n-1}\left(X, \Omega^{n}\right) \rightarrow H^{n-1}\left(D, \Omega^{n}\right)$ has dense image then the natural map $H_{2 n-1}(D, \mathbf{C}) \rightarrow H_{2 n-1}(X, \mathbf{C})$ is injective.

Proof. On $X$ we have the resolution of the constant sheaf $\mathbf{C}$ :

$$
0 \rightarrow \mathbf{C} \rightarrow \mathfrak{O} \stackrel{d}{\rightarrow} \Omega^{1} \stackrel{d}{\rightarrow} \cdots \stackrel{d}{\rightarrow} \Omega^{n} \rightarrow 0
$$

This resolution can be decomposed into short exact sequences:

$$
\begin{gathered}
0 \rightarrow \mathbf{C} \rightarrow \mathscr{O} \rightarrow \mathscr{F}_{1} \rightarrow 0 \\
0 \rightarrow \mathscr{F}_{1} \rightarrow \Omega^{1} \rightarrow \mathscr{F}_{2} \rightarrow 0 \\
\cdots \\
0 \rightarrow \mathscr{F}_{n-2} \rightarrow \Omega^{n-2} \rightarrow \mathscr{F}_{n-1} \rightarrow 0 \\
0 \rightarrow \mathscr{F}_{n-1} \rightarrow \Omega^{n-1} \rightarrow \Omega^{n} \rightarrow 0
\end{gathered}
$$

Since $X$ has no compact connected components it follows $H^{i}\left(X, \Omega^{j}\right)=0, j \geqslant 0$, $i \geqslant n\left(\Omega^{0}=\mathfrak{O}\right)$. So we get the isomorphisms:

$$
H^{n}\left(X, \mathscr{F}_{n-1}\right) \simeq \cdots \simeq H^{2 n-2}\left(X, \mathscr{F}_{1}\right) \simeq H^{2 n-1}(X, \mathbf{C})
$$

and the exact sequence:

$$
H^{n-1}\left(X, \Omega^{n}\right) \rightarrow H^{n}\left(X, \mathscr{F}_{n-1}\right) \rightarrow H^{n}\left(X, \Omega^{n-1}\right)=0
$$

It follows that the map $H^{n-1}\left(X, \Omega^{n}\right) \rightarrow H^{2 n-1}(X, \mathbf{C})$ is surjective. This map is 
defined as follows: if $\omega$ is an $(n, n-1)$ differential form $\bar{\partial}$-closed representing a cohomology class in $H^{n-1}\left(X, \Omega^{n}\right)$, then $\omega$ is also $d$-closed and therefore defines a cohomology class in $H^{2 n-1}(X, \mathbf{C})$. Similarly the map $H^{n-1}\left(D, \Omega^{n}\right) \rightarrow H^{2 n-1}$ $(D, \mathbf{C})$ is surjective. We have a commutative diagram of continuous maps

$$
\begin{array}{ccc}
H^{n-1}\left(X, \Omega^{n}\right) & \rightarrow & H^{2 n-1}(X, \mathbf{C}) \\
\downarrow & & \downarrow \\
H^{n-1}\left(D, \Omega^{n}\right) & \rightarrow & H^{2 n-1}(D, \mathbf{C}) .
\end{array}
$$

Hence, from the density of the map $H^{n-1}\left(X, \Omega^{n}\right) \rightarrow H^{n-1}\left(D, \Omega^{n}\right)$ it follows that the map $H^{2 n-1}(X, \mathbf{C}) \rightarrow H^{2 n-1}(D, \mathbf{C})$ has also a dense image. Since $H^{2 n-1}(X, \mathbf{C})$, $H^{2 n-1}(D, \mathbf{C})$ are Fréchet spaces whose topological duals are canonically isomorphic to $H_{2 n-1}(X, \mathbf{C})$ and $H_{2 n-1}(D, \mathbf{C})$ respectively [17], we get the desired conclusion.

Lemma 4. Let $X$ be a complex space of dimension $n, \pi: \tilde{X} \rightarrow X$ a resolution of singularities, $D \subset X$ an open subset and $\tilde{D}=\pi^{-1}(D)$. If the natural map $H_{2 n-1}(D, \mathbf{C})$ $\rightarrow H_{2 n-1}(X, \mathbf{C})$ is injective then the natural map $H_{2 n-1}(\tilde{D}, \mathbf{C}) \rightarrow H_{2 n-1}(\tilde{X}, \mathbf{C})$ is injective too.

Proof. Let $S=\operatorname{Sing}(X)$ and $\tilde{S}=\pi^{-1}(S)$. One has the following commutative diagram with exact lines:

$$
\begin{aligned}
& \cdots \rightarrow 0=H_{2 n-1}(\tilde{S}, \mathbf{C}) \rightarrow H_{2 n-1}(\tilde{X}, \mathbf{C}) \rightarrow H_{2 n-1}(\tilde{X}, \tilde{S} ; \mathbf{C}) \rightarrow H_{2 n-2}(\tilde{S}, \mathbf{C}) \rightarrow \cdots \\
& \downarrow \quad \downarrow \quad \downarrow 1 \downarrow \\
& \cdots \rightarrow 0=H_{2 n-1}(S, \mathbf{C}) \rightarrow H_{2 n-1}(X, \mathbf{C}) \rightarrow H_{2 n-1}(X, S ; \mathbf{C}) \rightarrow H_{2 n-2}(S, \mathbf{C}) \rightarrow \cdots
\end{aligned}
$$

$H_{2 n-1}(\tilde{S}, \mathbf{C})=H_{2 n-1}(S, \mathbf{C})=0$ since $\tilde{S}, S$ have complex dimension $\leq n-1$. Also, from the triangulation theorem for analytic sets [9] it follows that the map $H_{2 n-1}(\tilde{X}, \tilde{S} ; \mathbf{C}) \stackrel{\sim}{\rightarrow} H_{2 n-1}(X, S ; \mathbf{C})$ is an isomorphism. It follows that the map $H_{2 n-1}(\tilde{X}, \mathbf{C}) \rightarrow H_{2 n-1}(X, \mathbf{C})$ is injective. Similarly the map $H_{2 n-1}(\tilde{D}, \mathbf{C}) \rightarrow$ $H_{2 n-1}(D, \mathbf{C})$ is injective. If we consider the commutative diagram:

$$
\begin{array}{ccc}
H_{2 n-1}(\tilde{D}, \mathbf{C}) & \rightarrow & H_{2 n-1}(\tilde{X}, \mathbf{C}) \\
\downarrow & & \downarrow \\
H_{2 n-1}(D, \mathbf{C}) & \rightarrow & H_{2 n-1}(X, \mathbf{C})
\end{array}
$$

it follows that the map $H_{2 n-1}(\tilde{D}, \mathbf{C}) \rightarrow H_{2 n-1}(\tilde{X}, \mathbf{C})$ is injective, as desired.

In [15] the following result is proved: 
Lemma 5. Let $X$ be a complex manifold and $A \subset X$ a closed analytic subset. Then there exists $h \in C^{\infty}(X, \mathbf{R})$ such that:

1) $h \geqslant 0, A=\{h=0\}$

2) for any point $x \in X$ there is an open neighbourhood $U=U(x)$ of $x$ and $\theta$ $\in C^{\infty}(U, \mathbf{R})$ such that $\log \left(\left.h\right|_{U \backslash A}\right)+\left.\theta\right|_{U \backslash A}$ is 1 -convex. We shall need also:

Lemma 6. Let $Y$ be a complex space of dimension $k, K \subset Y$ a compact subset and $D \subset Y$ an open subset such that $K \subset D \subset Y$. Then there exists $a(k+1)$ convex exhaustion function $\phi: Y \rightarrow \mathbf{R}$ such that $K \subset\{\phi<0\}$ and $\{\phi \leqslant 0\} \subset D$.

Proof. It is clear that for smooth $Y$ it is nothing to prove. For the general case the proof is by induction on $\operatorname{dim}(Y)$.

If $\operatorname{dim}(Y)=0$ the statement of the lemma is obvious. So we assume that the statement of Lemma 6 holds for complex spaces of dimension $\leqslant k-1$. If $Y$ has dimension $k$ then $Z=\operatorname{Sing}(Y)$ has dimension $\leqslant k-1$ and, by the induction hypothesis, there exists a $k$-convex exhaustion function $\varphi$ on $Z$ with $\varphi<0$ on $K \cap Z$ and $\{\varphi \leqslant 0\} \subset D \cap Z$. By Lemma 1 and Corollary 2 we may assume that $\varphi$ can be extended to a $k$-convex function $\tilde{\varphi}$ in a neighbourhood of $Z$ in $Y$ (replacing $\varphi$ by an approximation of it). Modifying suitably this function outside a neighbourhood of $Z$ we get the desired function on $Y$ (noting, of course, that the $(k+1)$-convexity means nothing on $\operatorname{Reg}(Y))$.

The proof of Lemma 6 complete.

LEMMA 7. Let $X$ be a complex manifold of pure dimension $n$ with no compact connected components, let $A \subset X$ be a closed analytic subset and $K \subset X$ a compact subset such that $X \backslash K$ has no relatively compact connected components. Let $V$ be any open neighbourhood of $A \cup K$. Then there exists a function $\varphi: X \rightarrow[-\infty, \infty)$ with the following properties:

1) $A=\{\varphi=-\infty\}, \exp \varphi$ is smooth, $\left.\varphi\right|_{X \backslash A}$ is n-convex

2) $A \cup K \subset\{\varphi<0\}$ and $\{\varphi \leqslant 0\} \subset V$

3) $\left.\varphi\right|_{X \backslash V}$ is an exhaustion function

Proof. By Lemma 2 there exists a $n$-convex exhaustion function $\phi: X \rightarrow \mathbf{R}$ such that $K \subset\{\phi<0\}$ and $\{\phi \leqslant 0\} \subset V$. Let also $h \in C^{\infty}(X, \mathbf{R})$ be a function with the properties stated in Lemma 5 . Then it is easy to see that one can take $\varphi=\chi \circ \phi+\log h$ where $\chi: \mathbf{R} \rightarrow \mathbf{R}$ is a suitable strictly increasing smooth convex function with $\chi(t)=A t$ if $t \leqslant 0$ and $A>0$ is sufficiently large.

We can now prove the main lemma needed in the proof of the Theorem: 
Lemma 8. Let $X$ be a complex space of pure dimension $n$ with no compact irreducible components. Let also $\pi: \tilde{X} \rightarrow X$ be a resolution of singularities, $D \subset X$ an open subset and $\tilde{D}=\pi^{-1}(D)$. If $\tilde{X} \backslash \tilde{D}$ has no compact connected components then $D$ is $n$-Runge in $X$.

Proof. Let $S=\operatorname{Sing}(X)$ and $\tilde{S}=\pi^{-1}(S)$, hence $\pi: \tilde{X} \backslash \tilde{S} \rightarrow X \backslash S$ is an isomorphism. Let $K \subset D$ be any compact subset. We have to find a $n$-convex exhaustion function $\varphi: X \rightarrow \mathbf{R}$ such that $K \subset\{\varphi<0\}$ and $\{\varphi \leqslant 0\} \subset D$. Let $Q_{1}=$ $\pi^{-1}(K) \subset \tilde{D}$ and define $Q$ to be the union of $Q_{1}$ and of those components of $\tilde{X} \backslash Q_{1}$ which are relatively compact in $\tilde{X}$. Then $Q$ is a compact set and $Q \subset \tilde{D}$ because $\tilde{X} \backslash \tilde{D}$ has no compact connected components. We set $K^{\prime}=\pi(Q)$ and $L=K^{\prime} \cap S$. By Lemma 6 for the inclusion $L \subset D \cap S \subset S$ we can find a $n$-convex exhaustion function $\varphi_{1}: S \rightarrow \mathbf{R}$ such that $L \subset\left\{\varphi_{1}<0\right\}$ and $\left\{\varphi_{1} \leqslant 0\right\} \subset D \cap S$. By Lemma 1 and Corollary 2 we may assume that $\varphi_{1}$ can be extended to an $n$-convex function $\varphi_{2}$ defined in an open neighbourhood $W$ of $S$ (replacing $\varphi_{1}$ by an approximation of it). Let $V_{1}$ be a sufficiently small open neighbourhood of $S, \bar{V}_{1} \subset W$, such that $\varphi_{2}$ $\left.\right|_{\bar{V}_{1}}$ is an exhaustion function, $\bar{V}_{1} \cap\left\{\varphi_{2} \geq-\varepsilon_{0}\right\} \cap K^{\prime}=\phi$ for some $\varepsilon_{0}>0$ and $\bar{V}_{1}$ $\cap\left\{\varphi_{2} \leqslant 0\right\} \subset D$. Let $V_{2}$ be an open neighbourhood of $S$ with $\bar{V}_{2} \subset V_{1}$. We choose now an open neighbourhood $U$ of $K^{\prime}, U \subset \subset D, \bar{U} \cap \bar{V}_{1} \cap\left\{\varphi_{2} \geqslant-\varepsilon_{0}\right\}=\phi$ and we define $H=U \cup V_{2}$ and $V=\pi^{-1}(H) \subset \tilde{X}$. Then $V$ is an open neighbourhood of $\tilde{S} \cup Q$ and using Lemma 7 for $\tilde{X}, Q, \tilde{S}(\tilde{X} \backslash Q$ has no connected components which are relatively compact in $\tilde{X})$ we obtain a function $\phi: X \rightarrow[-\infty$, $\infty$ ) (induced by the isomorphism $\pi: \tilde{X} \backslash \tilde{S} \rightarrow X \backslash S$ ) such that: $S=\{\phi=-\infty\}$, $\exp \phi$ is continuous, $\phi$ is $n$-convex outside $S, S \cup K^{\prime} \subset\{\phi<0\},\{\phi \leqslant 0\} \subset H$ and $\left.\phi\right|_{X \backslash H}$ is an exhaustion function. In particular $\left.\phi\right|_{\bar{V}_{1} \backslash V_{2}}$ is also an exhaustion function. Moreover, on the set $\left(\bar{V}_{1} \backslash V_{2}\right) \cap\{\phi \leqslant 0\}$ we have $\varphi_{2} \leqslant-\varepsilon_{0}$. It follows that we can find a smooth strictly increasing convex function $\theta: \mathbf{R} \rightarrow \mathbf{R}, \theta(t)=$ $\alpha t$ if $t \leqslant 0\left(\alpha>0\right.$ small enough) such that setting $\phi_{1}=\theta^{\circ} \psi$ one has $\phi_{1}>\varphi_{2}$ on $\bar{V}_{1} \backslash V_{2}$ and $\left.\phi_{1}\right|_{X \backslash H}$ is an exhaustion function. Clearly $\phi_{1}(x) \rightarrow-\infty$ if $x \rightarrow y \in S$.

We define now a function $\beta: X \rightarrow \mathbf{R}$ which is locally the maximum of two $n$-convex functions, hence $\beta \in F_{n}(X)$, in the following way: If $x \in V_{1}$ we put $\beta(x)=\max \left(\varphi_{2}(x), \phi_{1}(x)\right)$. In a neighbourhood of $S \beta$ is equal to $\varphi_{2}$ since $\phi_{1}(x) \rightarrow-\infty$ if $x \rightarrow y \in S$. Since $\varphi_{2}$ is $n$-convex on $V_{1}$ and $\phi_{1}$ is $n$-convex outside $S$ it follows that on $V_{1} \beta$ is locally the maximum of two $n$-convex functions.

If $x \in X \backslash \bar{V}_{2}$ we define $\beta(x)=\phi_{1}(x)$. On $V_{1} \backslash \bar{V}_{2}$ the two definitions of $\beta$ agree because $\phi_{1}(x)>\varphi_{2}(x)$ if $x \in V_{1} \backslash \bar{V}_{2}$. Hence $\beta \in F_{n}(X)$. Since $\left.\varphi_{2}\right|_{\bar{V}_{1}}$ is an exhaustion function and $\left.\phi_{1}\right|_{X \backslash H}$ is an exhaustion function it follows that $\beta$ is an exhaustion function on $X$. Also one has $\{\beta \leqslant 0\} \subset \bar{U} \cup\left[\bar{V}_{1} \cap\left\{\varphi_{2} \leqslant 0\right\}\right] \subset D$, 
hence $\{\beta \leqslant 0\}$ is a compact subset of $D$. From the definition of $\beta$ it follows that $K \subset K^{\prime} \subset\{\beta<0\}$. Lemma 8 follows now from Corollary 2 because $\beta \in F_{n}(X)$.

To prove the Theorem we need also some simple topological lemmas.

In [12] the following topological result is proved:

Lemma 9. Let $M$ be a locally compact Hausdorff space, $A \subset M$ a closed subset and $P$ a connected compact component of $A$. Then there exists a fundamental system of open neighbourhoods $V$ of $P$ in $M$ such that $(\partial V) \cap A=\phi$ where $\partial V$ denotes the boundary of $V$ in $M$.

From this result it follows immediately:

Lemma 10. Let $M$ be a locally compact Hausdorff space and $A \subset M$ a closed subset. Then $A$ has compact connected components iff $A$ can be written $A=L \cup F$ where $L \neq \phi$ is a compact set, $F$ is a closed set and $L \cap F=\phi$.

Proof. If $A$ has a compact connected component $P$, then by Lemma 9 there is an open neighbourhood $V$ of $P$ with $\bar{V}=$ compact and $(\partial V) \cap A=\phi$. We define then $L=\bar{V} \cap A$ and $F=(\mathrm{C} V) \cap A$.

Conversely, if $A$ can be written $A=L \cup F$ with $L \neq \phi$ compact set and $F$ closed set, $L \cap F=\phi$, we choose a connected component $P$ of $A$ with $P \cap L \neq \phi$. From the equality $P=(P \cap L) \cup(P \cap F)$ it follows $P \subset L$ and therefore $P$ is a compact connected component of $A$.

Lemma 11. Let $X, Y$ be locally compact Hausdorff spaces and $\pi: X \rightarrow Y$ a continuous map which is proper and surjective. Let $A \subset Y$ be a closed subset and $\tilde{A}=$ $\pi^{-1}(A)$. The following statements hold:

1) If $\tilde{A}$ has no compact connected components then $A$ has no compact connected components.

2) Assume additionally that $\pi$ has connected fibers. Then the condition " $A$ has no compact connected components" implies " $\tilde{A}$ has no compact connected components".

Proof. 1) follows immediately by Lemma 10 . So let us verify 2). Assume that $\tilde{A}$ has compact connected components. Then by Lemma $10 \tilde{A}$ can be written $\tilde{A}=$ $L \cup F$ with $L \neq \phi$ compact set, $F$ closed set and $L \cap F=\phi$. Therefore $A=$ $\pi(L) \cup \pi(F), \pi(\Gamma) \neq \phi$ is a compact set and $\pi(F)$ is a closed set. Since $\pi$ has connected fibers it follows easily that $\pi(L) \cap \pi(F)=\phi$, and by Lemma $10 \mathrm{~A}$ has compact connected components. The proof of Lemma 11 is complete. 
Lemma 12. Let $X$ be a complex manifold of pure dimension $n$ with no compact connected components and let $D \subset X$ be an open subset. Then the natural map $H_{2 n-1}(D, \mathbf{C}) \rightarrow H_{2 n-1}(X, \mathbf{C})$ is injective iff $X \backslash D$ has no compact connected components.

Proof. We have the exact sequence:

$$
\cdots \rightarrow H_{2 n}(X, \mathbf{C}) \rightarrow H_{2 n}(X, D ; \mathbf{C}) \rightarrow H_{2 n-1}(D, \mathbf{C}) \rightarrow H_{2 n-1}(X, \mathbf{C}) \rightarrow \cdots
$$

Since $X$ has no compact connected components it follows that $H_{2 n}(X, \mathbf{C})=0$, therefore the injectivity of the map $H_{2 n-1}(D, \mathbf{C}) \rightarrow H_{2 n-1}(X, \mathbf{C})$ is equivalent to the vanishing of the relative homology group $H_{2 n}(X, D ; \mathbf{C})$. By Alexander duality [18] we have: $H_{2 n}(X, D ; \mathbf{C}) \cong \bar{H}_{c}^{0}(X \backslash D, \mathbf{C})=\{f: X \backslash D \rightarrow \mathbf{C} \mid f$ is locally constant and $f=0$ outside a compact subset of $X \backslash D$ \}. Using this isomorphism Lemma 12 is a direct consequence of Lemma 10.

Lemma 13. Let $X$ be a pure dimensional complex space, $\pi: \tilde{X} \rightarrow X$ a resolution of singularities, $D \subset X$ an open subset and $\tilde{D}=\pi^{-1}(D)$. Then the following conditions are equivalent:

1) $X \backslash D$ has no compact irreducible components

2) $\tilde{X} \backslash \tilde{D}$ has has no compact connected components

Proof. Let $\nu: \hat{X} \rightarrow X$ be the normalization of $X$ and $\hat{D}=\nu^{-1}(D)$. By definition $X \backslash D$ has no compact irreducible components iff $\hat{X} \backslash \hat{D}$ has no compact connected components. Consider the commutative diagram:

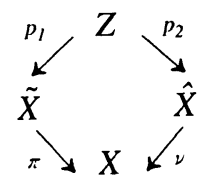

where $Z=\tilde{X} \times_{X} \hat{X}$ is the reduced fiber product of $\tilde{X}$ and $\hat{X}$ over $X$ and $p_{1}, p_{2}$ are the canonical projections given by $p_{1}=\tilde{p}_{1} \circ i$ and $p_{2}=\tilde{p}_{2} \circ$. Here $\tilde{p}_{1}: \tilde{X} \times \hat{X} \rightarrow \tilde{X}$ is the projection on $\tilde{X}, \tilde{p}_{2}: \tilde{X} \times \hat{X} \rightarrow \hat{X}$ is the projection on $\hat{X}$ and $i: Z \rightarrow \tilde{X} \times \hat{X}$ is the inclusion map. $Z$ is the reduced subspace given by $Z=\{(\tilde{x}, \hat{x}) \in \tilde{X} \times \hat{X}$ $\mid \pi(\tilde{x})=\nu(\hat{x})\}$. It is easy to see that $p_{1}$ and $p_{2}$ are proper modifications. Moreover, since $\tilde{X}$ and $\hat{X}$ are normal complex spaces, it follows from the Riemann extension theorem that $p_{1 *} \mathscr{O}_{Z} \cong \mathscr{O}_{\tilde{X}}$ and $p_{2 *} \mathscr{O}_{Z} \cong \mathscr{O}_{\widehat{X}}$. in particular $p_{1}$ and $p_{2}$ have connected fibers. From these remarks Lemma 13 is a direct consequence of Lemma 11. 
Proof of the Theorem.

1) $\Rightarrow$ 2) follows from the paper of Andreotti and Grauert [1].

2) $\Rightarrow$ 3) follows from the coherence of $\Omega_{X}^{n}$

3) $\Rightarrow 5$ ) Let $\pi: \tilde{X} \rightarrow X$ be a resolution of singularities and $\tilde{D}=\pi^{-1}(D)$. Consider the commutative diagram:

$$
\begin{array}{ccc}
H^{n-1}\left(\tilde{X}, \Omega_{\tilde{X}}^{n}\right) & \stackrel{r_{1}}{\longrightarrow} & H_{n-1}\left(\tilde{D}, \Omega_{\tilde{X}}^{n}\right) \\
\alpha \uparrow & & \uparrow \beta \\
H^{n-1}\left(X, \Omega_{X}^{n}\right) & \stackrel{r_{2}}{\longrightarrow} & H_{n-1}\left(D, \Omega_{X}^{n}\right)
\end{array}
$$

where $\alpha, \beta, r_{1}, r_{2}$ are continuous maps and $r_{2}$ has dense image by our hypothesis. By Theorem 1 the maps $\alpha$ and $\beta$ are bijective, hence $r_{2}$ has also dense image. By Lemma 3 we get the injectivity of the map $H_{2 n-1}(\tilde{D}, \mathbf{C}) \rightarrow H_{2 n-1}(\tilde{X}, \mathbf{C})$ and by Lemma $12 \quad \tilde{X} \backslash \tilde{D}$ has no compact connected components. In view of Lemma 13 $X \backslash D$ has no compact irreducible components.

$5) \Rightarrow 1$ ) If $\pi: \tilde{X} \rightarrow X$ is a resolution of singularities and $\tilde{D}=\pi^{-1}(D)$ it follows from Lemma 13 that $\tilde{X} \backslash \tilde{D}$ has no compact connected components and by Lemma $8 D$ is $n$-Runge in $X$.

Therefore we have proved 1$) \Leftrightarrow 2) \Leftrightarrow 3) \Leftrightarrow 5$ ).

1) $\Rightarrow$ 4) follows from Corollary 1 .

4) $\Rightarrow 5$ ) Let $\pi: \tilde{X} \rightarrow X$ be a resolution of singularities and $\tilde{D}=\pi^{-1}(D)$. By Lemma 4 the natural map $H_{2 n-1}(\tilde{D}, \mathbf{C}) \rightarrow H_{2 n-1}(\tilde{X}, \mathbf{C})$ is injective and by Lemma $12 \tilde{X} \backslash \tilde{D}$ has no compact connected components. In view of Lemma 13 $X \backslash D$ has no compact irreducible components.

The proof of the Theorem is complete.

Remark. One can easily see that in the Theorem the condition " $X$ has pure dimension $n$ and has no compact irreducible components" can be weakened to " $X$ has dimension $n$ and has no compact irreducible components of dimension $n$ ". In this case the condition 5) must be replaced by

$\left.5^{\prime}\right) \quad X \backslash D$ has no compact irreducible components of dimension $n$. This means the following: if $\nu: \hat{X} \rightarrow X$ is the normalization map and $\hat{D}=\nu^{-1}(D)$ then $\hat{X}_{i} \backslash \hat{D}$ has no compact connected components for every $n$-dimensional connected component $\hat{X}_{i}$ of $\hat{X}$.

Acknowledgement. This paper was written while the first author was a visitor at the University of Rome "La Sapienza" and at the University of Florence. He would like to thank CNR and the departments of mathematics "Guido Castelnuovo" and "Ulisse Dini" for the given support. 


\section{REFERENCES}

[1] A. Andreotti and H. Grauert, Théorèmes de finitude pour la cohomologie des éspaces complexes, Bull. Soc. Math. France, 90 (1962), 193-259.

[2] H. Behnke and K. Stein, Entwicklungen analytischer Funktionen auf Riemannsche Flachen. Math. Ann., 120 (1948), 430-461.

[3] M. Colţoiu, $n$-concavity of $n$-dimensional complex spaces, Math. Z., 210 (1992), $203-206$

[4] J. P. Demailly, Cohomology of $q$-convex spaces in top degrees, Math. Z., 204 (1990), $283-295$

[ 5 ] K. Diederich and J. E. Fornaess, Smoothing $q$-convex functions in the singular case, Math. Ann., 273 (1986), 665-671.

[6] H. Grauert and O. Riemenschneider, Verschwindungssätze für analytische Kohomologiegruppen auf komplexen Räume, Invent. Math., 11 (1970), 263-292.

[7] R. E. Greene and H. Wu. Embedding of open riemannian manifolds by harmonic functions, Ann. Inst. Fourier, 25 (1975), 215-235.

[8] H. Hironaka, Desingularization of complex-analytic varieties, Actes Congrès Int. Math., 2 (1970), 627-631.

[9] S. Kojasiewicz, Triangulation of semi-analytic sets, Ann. Scuola Norm. Sup. Pisa., 18 (1964), 449-474.

[10] G. Lupacciolu, Topological properties of $q$-convex sets, Trans, Amer. Math. Soc., 337 (1993), 427-435.

[11] N. Mihalache, The Runge theorem on 1-dimensional Stein spaces, Rev. Roumaine Math. Pures et Appl., 33 (1988), 7, 601-611.

[12] R. Narasimhan, Complex analysis in one variable, Birkhäuser, 1985.

[13] T. Ohsawa, Completeness of non-compact analytic spaces, Publ. Res. Inst. Math. Sci., 20 (1984), 683-692.

[14] - A vanishing theorem for proper direct images, Publ. Res. Inst. Math. Sci., 23 (1987), 243-250.

[15] M. Peternell, Algebraische Varietäten und $q$-vollständige komplexe Räume, Math. Z., 200 (1989), 547-581.

[16] A. Silva, Behnke-Stein theorem for analytic spaces, Trans. Amer. Math. Soc., 199 (1974), 317-326.

[17] G. Sorani, Homologie des $q$-paires de Runge, Ann. Scuola Norm. Sup. Pisa, 17 (1963), 319-332.

[18] E. H. Spanier, Algebraic topology, Mc Graw Hill, 1966.

[19] K. Takegoshi, Relative vanishing theorems in analytic spaces, Duke Math. J., 52 (1985), 273-279.

[20] V. Vâjâitu, Approximation theorems and homology of $q$-Runge domains in complex spaces, to appear in J. reine angew. Math., 1994.

[21] B. M. Weinstock, An approximation theorem for $\bar{\partial}$-closed forms of type $(n, n-1)$, Proc. Amer. Math. Soc., 26 (1970), 625-628. 
Mihnea Colţoiu

Institute of Mathematics of

the Romanian Academy

P. O. Box 1-764 RO-70700 Bucharest

Romania

Alessandro Silva

Dipartimento di Matematica

"G. Castelnuovo"

Università di Roma "La Sapienza"

I-00185 Roma

Italy 